Supporting Information

\title{
Printable Liquid Metal Microparticle Ink for Ultrastretchable Electronics
}

Yanyan Li, ${ }^{1}$ Shuxuan Feng, ${ }^{1}$ Shitai Cao, ${ }^{1}$ Jiaxue Zhang, ${ }^{1}$ Desheng Kong ${ }^{1 *}$

${ }^{1}$ College of Engineering and Applied Sciences, State Key Laboratory of Analytical

Chemistry for Life Science, National Laboratory of Solid State Microstructure, Collaborative Innovation Center of Advanced Microstructures, and Jiangsu Key

Laboratory of Artificial Functional Materials, Nanjing University, Nanjing 210093, China.

*Corresponding author: dskong@nju.edu.cn (D.K.). 


\section{Supporting Figures}

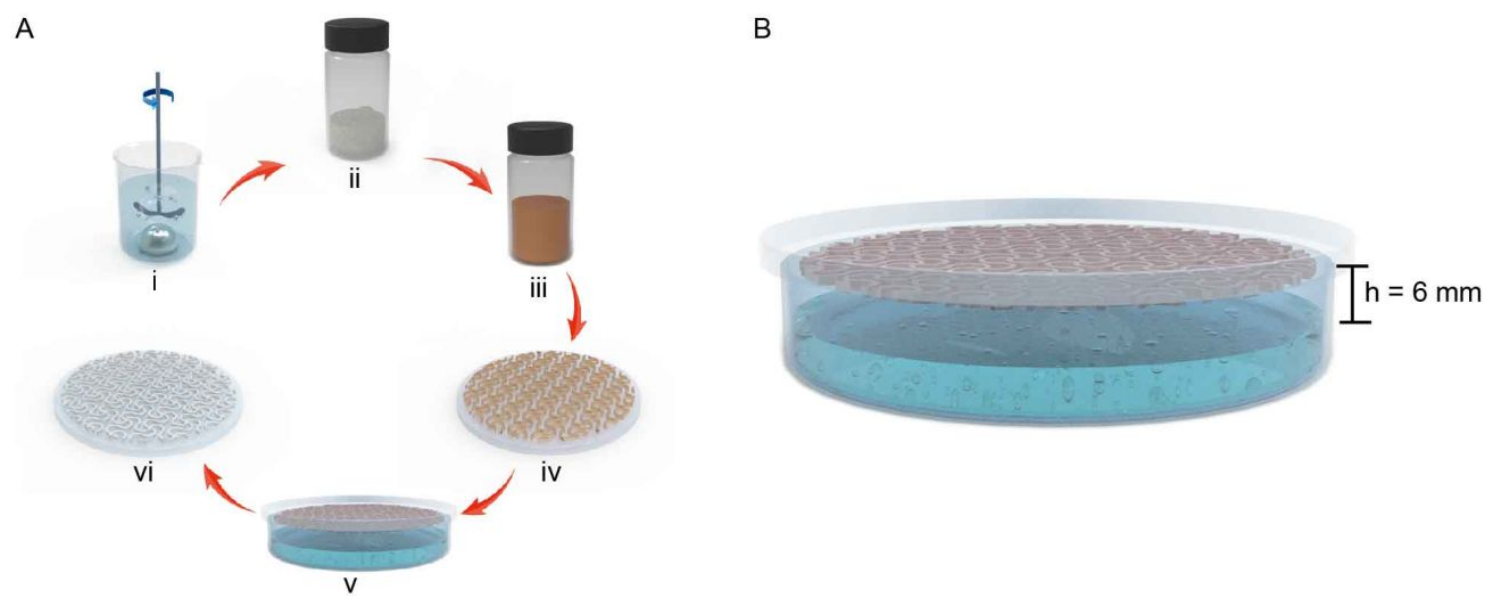

Figure S1. Schematic illustration of the overall process flow and the chemical sintering procedure. (A) Step-by-step procedures to create ultrastretchable conductor involving the synthesis of liquid metal microparticles $(i$, ii), formulation of printable ink (iii), screen-printing of various features (iv), chemical sintering of the printed composite (v), and the formation of sintered conductor (vi). (B) Experimental set-up for the chemical sintering process. A $10 \mathrm{~cm}$ glass dish is filled with concentrated hydrochloride solution. As-printed composites are exposed to acid fume by facing downward at $\sim 6 \mathrm{~mm}$ above the surface of the solution.
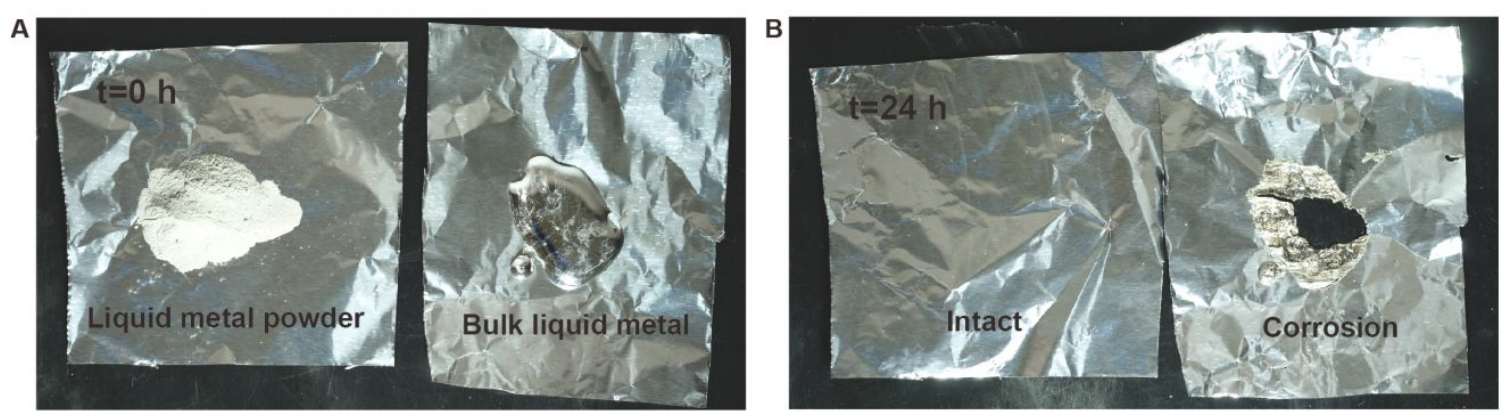


\section{Figure S2. Corrosion characteristics of powder and bulk forms of liquid metal}

towards Al foils. (A) Optical image of liquid metal microparticles (left) and bulk liquid metal (right) placed on aluminum foils. (B) Optical image of the aluminum foils after storage under the ambient conditions for $24 \mathrm{~h}$, acquired by washing away liquid metal with ethanol. A large hole formed on the Al foil reveals the corrosion effect by bulk liquid metal. In contrast, the Al foil remains intact without visible damage after storing liquid metal microparticles. The surface oxides on these microparticles effectively passivate the active liquid metal cores to suppress the corrosion process.
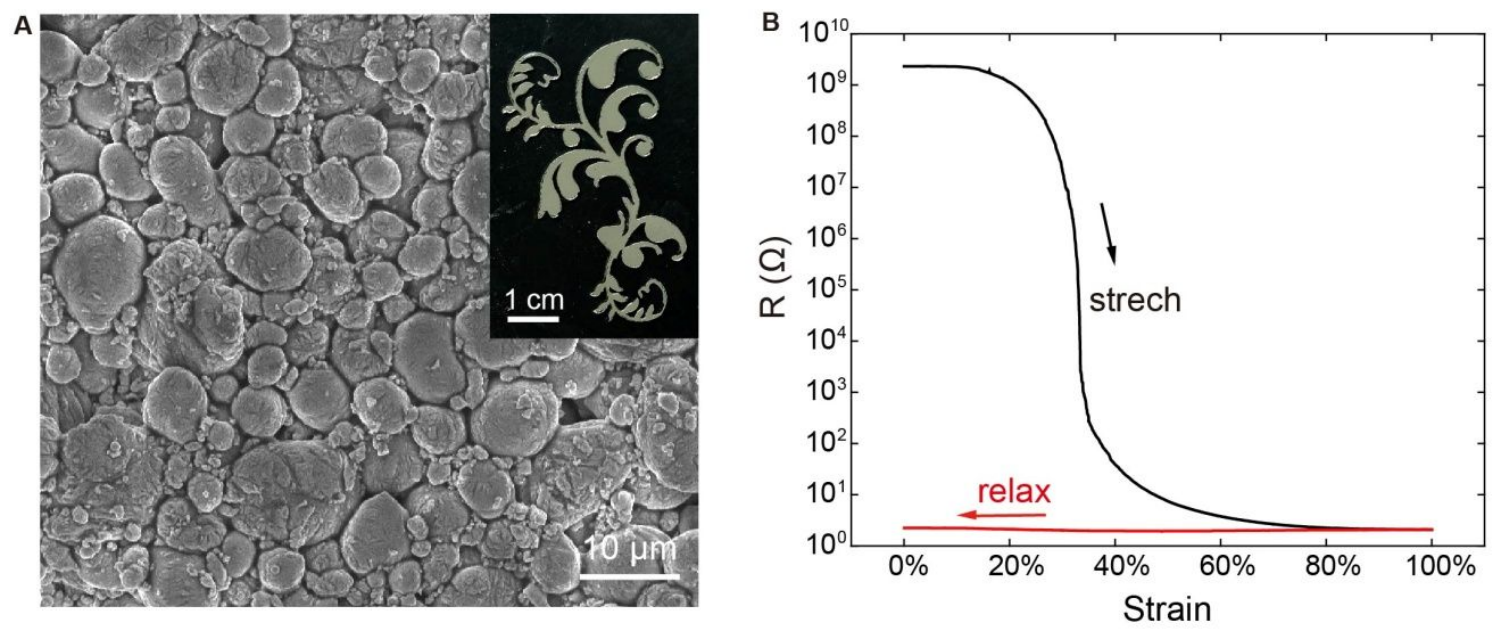

Figure S3. Characterizations on printed liquid metal microparticle/SIS composites. (A) SEM image to reveal the microstructure of the printed composite consisting of isolated microparticles. Inset: Optical image of a flower-shaped pattern on a SIS substrate. (B) Conductance as a function of the tensile strain upon uniaxial stretching and relaxation. The sharp increase in conductivity at $\sim 30 \%$ strain signifies the coalescence of liquid metal microparticles. After mechanical activation at $100 \%$ strain, the conductivity of sintered conductor is $\sim 410 \mathrm{~S} \mathrm{~cm}^{-1}$ at the relaxed state. The relatively low conductivity is largely associated with the insufficient transfer of mechanical forces by the extremely compliant and deformable substrate. 

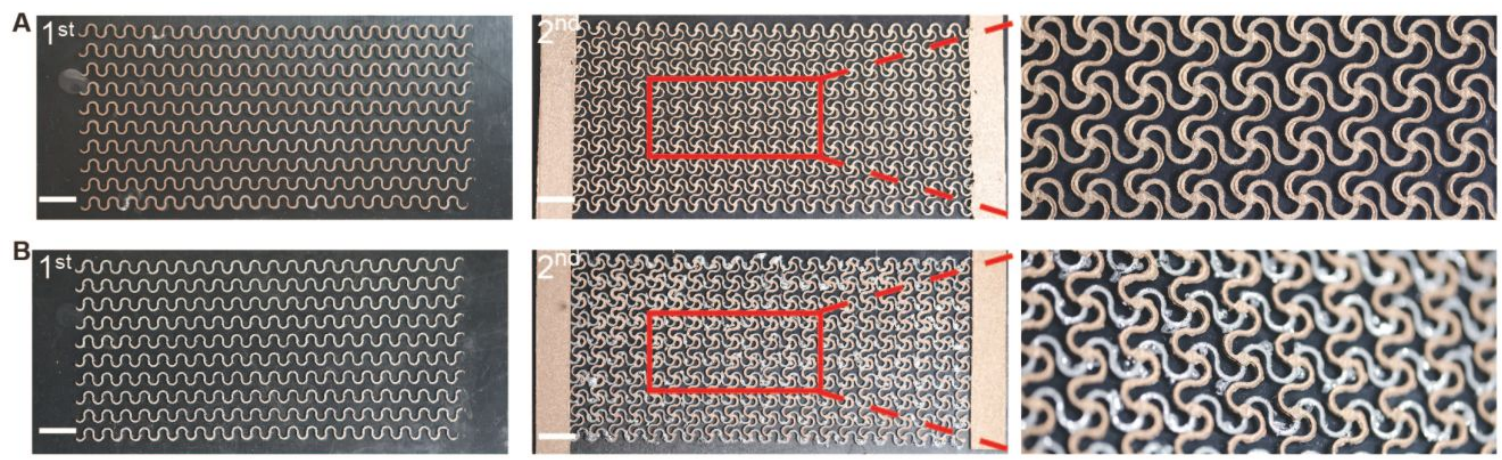

Figure S4. Optical images of a complex pattern of stretchable heater generated by a two-step printing process. (A) Heater pattern by two sequential printing steps. (B) Heater pattern formed by chemical sintering of the first layer and subsequent printing of the second layer. Scale bars: $1 \mathrm{~cm}$. The solid-state nature of as-deposited features provides the basis for the use of multiple printing steps to define complex patterns. In contrast, additional printing steps are challenging to perform after the chemical sintering process, which recovers the liquid state properties for printed composites. The original printed patterns are easily deformed as a result of the scratches caused by the stencil mask.
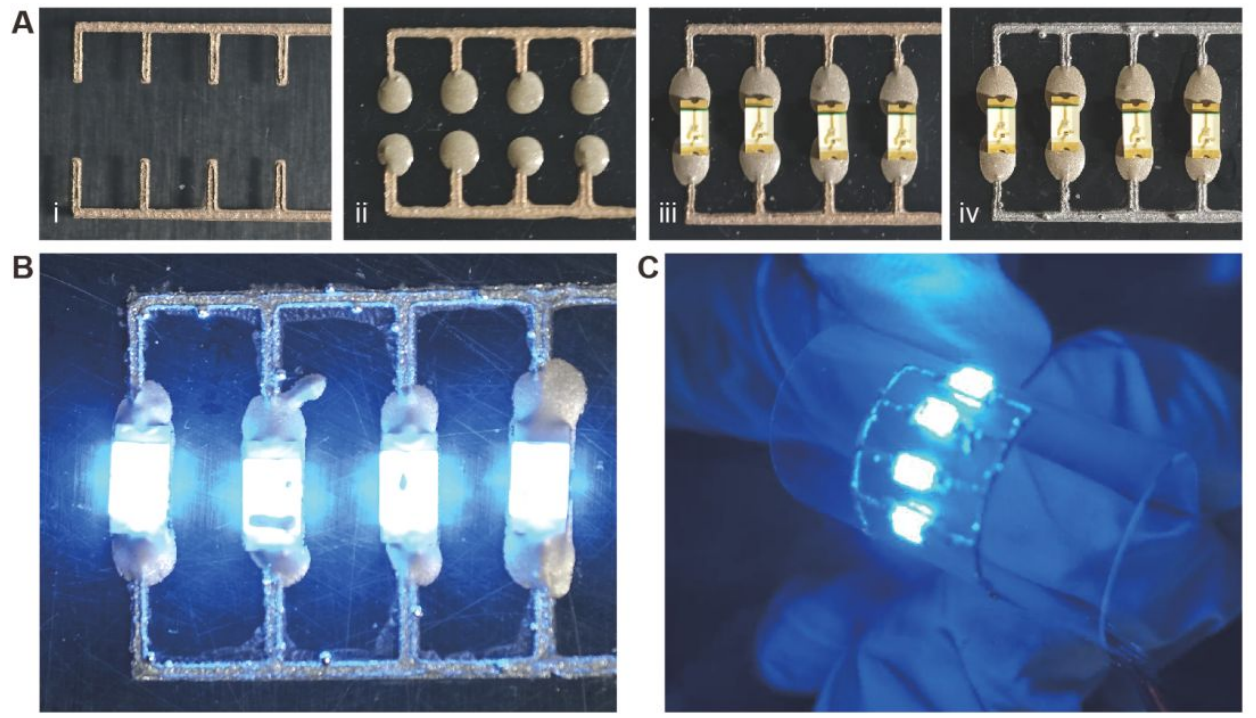

Figure S5. Optical images to illustrate the flexible combination of printed liquid metal with printed silver composite. (A) Optical images to illustrate the step-by-step procedures for a flexible LED array, including interconnect pattern of printed liquid 
metal composite on a PET substrate (i), printed contact pads by conductive silver ink (Nanjing Helite Adhesive Co., Ltd.) (ii), LED chips (LTST-C150ZBKT-5A, 1206, peak emission at $468 \mathrm{~nm}$, Lite-On Inc.) mounted on the contact pads (iii), and post treatment by chemical sintering process (iv). (B) Image showing the operation of the LED array. (C) LED array working under bent state to reveal its flexibility. Chemical sintering process coverts solid-state liquid metal composite into deformable conductor interconnected with conductive silver composite. Printable liquid metal ink developed here is well-suited for a wide range of applications in flexible electronics.

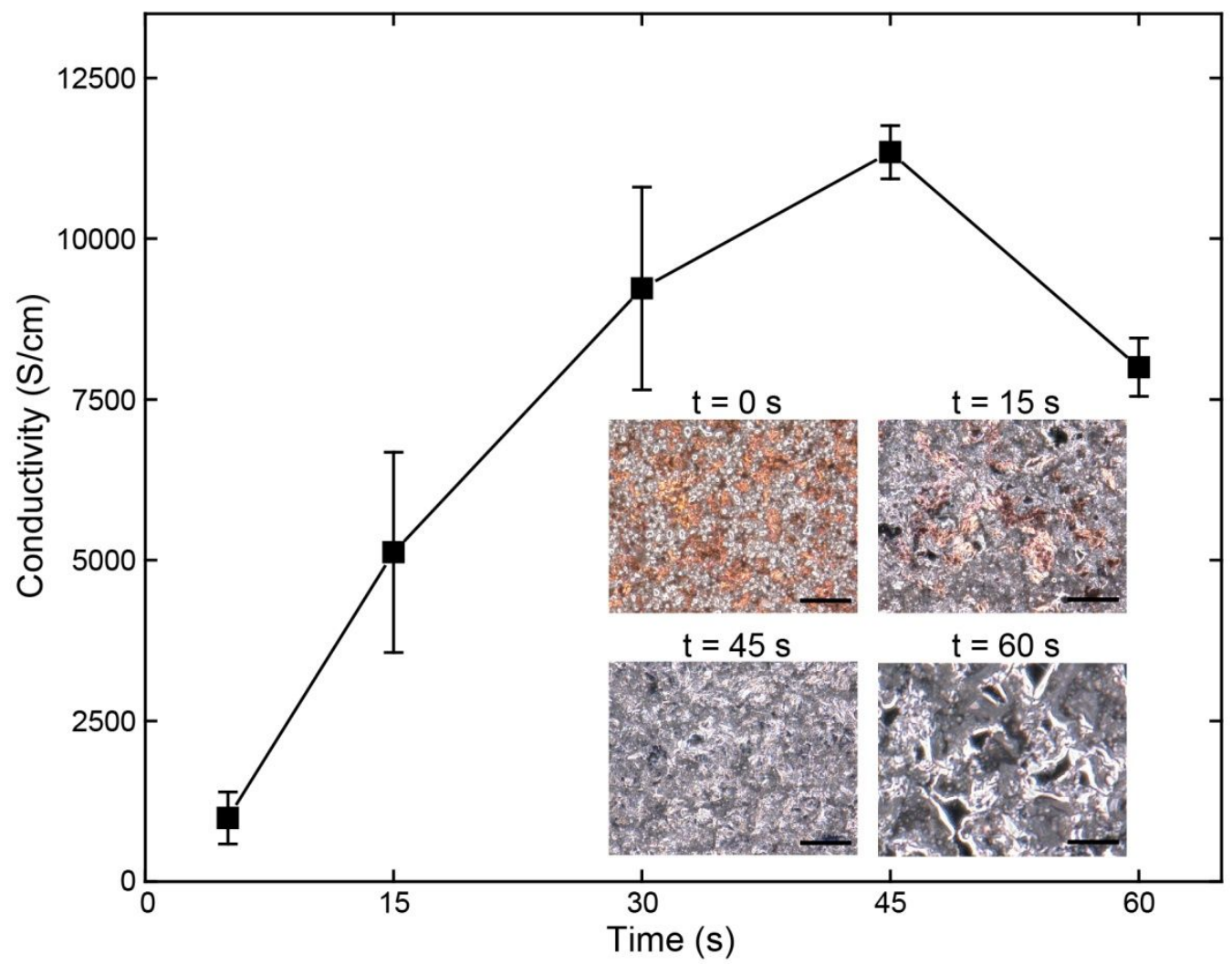

Figure S6. Influence of the processing duration in hydrochloric acid fume on the conductivity of printed liquid metal microparticle/Cu nanoflake/SIS composite $(\phi=$ 0.1). Inset: Optical microscope images to reveal the evolution of the microstructure and the color during the sintering process. Scale bars are $50 \mu \mathrm{m}$. A short exposure for $5 \mathrm{~s}$ is sufficient to establish the metallic conductivity of $990 \mathrm{~S} \mathrm{~cm}^{-1}$. The increase in processing time allows additional liquid metal droplets to bind with $\mathrm{Cu}$ nanoflakes, which gives rise to marked improvement in electrical conductivity. The extended exposure, on the other hand, has adverse effects on sintered conductor due to the corrosion issue. The optimal duration for chemical sintering process is determined as $45 \mathrm{~s}$. 


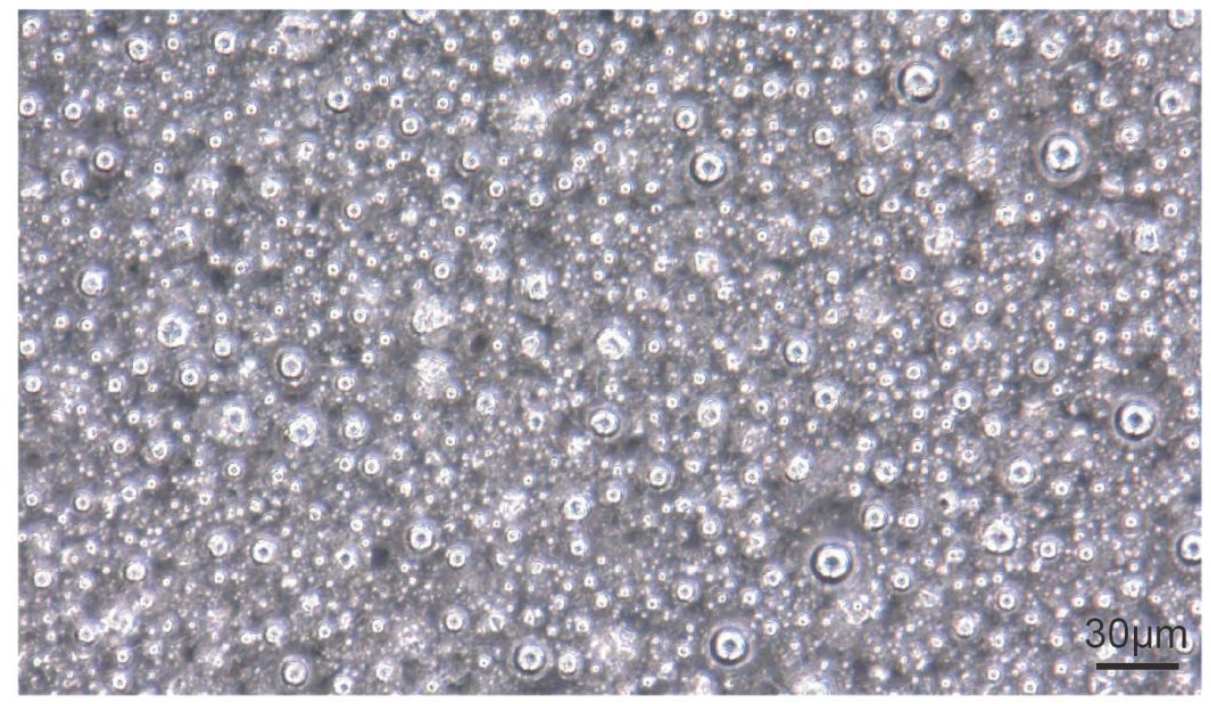

Figure S7. Optical microscopy image of liquid metal particles/SIS composite after the treatment in acid fume. The coalescence of liquid metal microparticles into isolated beads inhibits the formation of conductive network. 


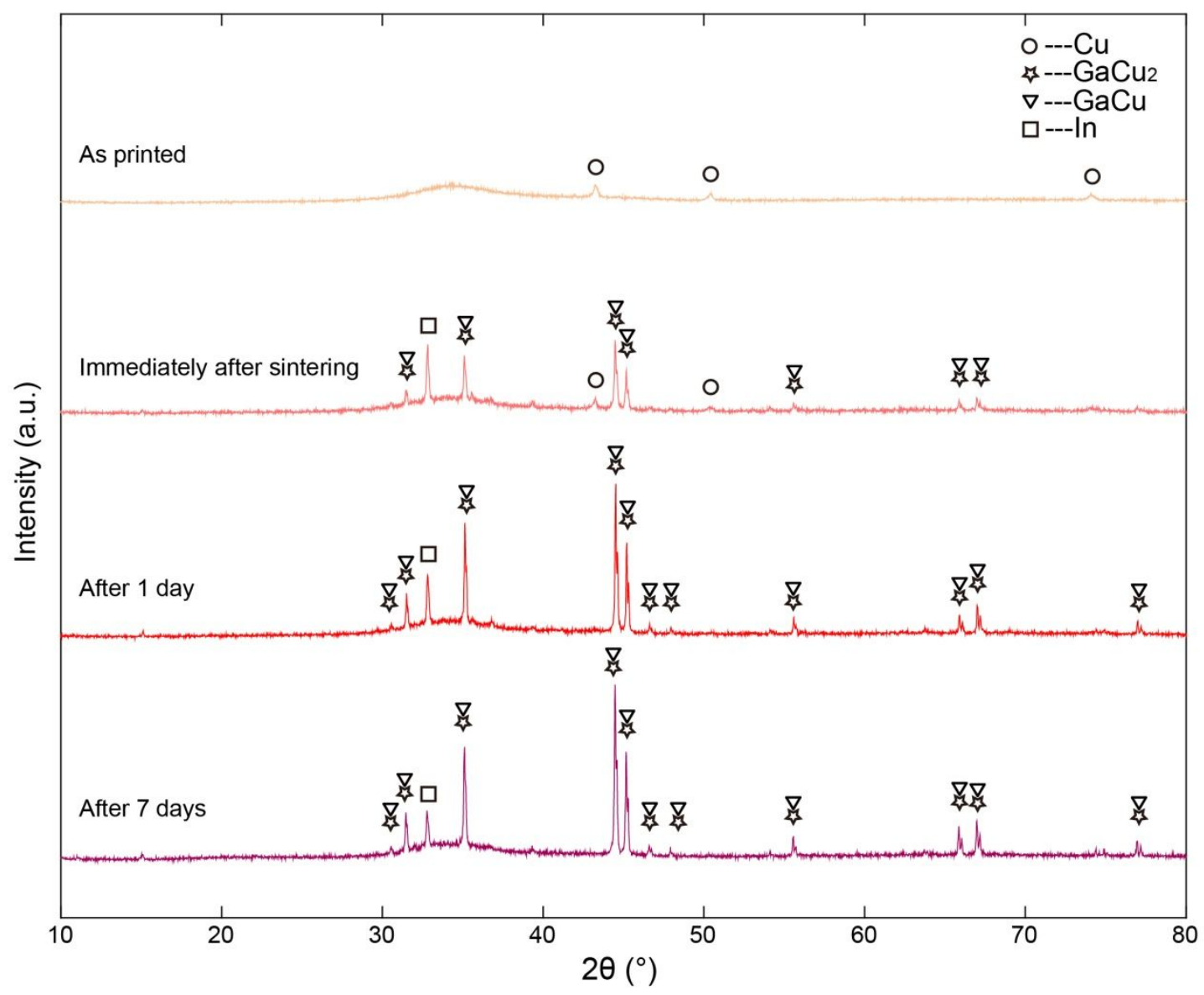

Figure S8. X-ray diffraction spectra of the printed composite $(\phi=0.1)$. Crystalline peaks for intermetallic compounds of $\mathrm{GaCu}_{2}$ and $\mathrm{GaCu}$ are readily identified immediately after the chemical treatment in acid fume. These peaks grow and saturate within one day, which suggest the completion of the alloying reaction. 

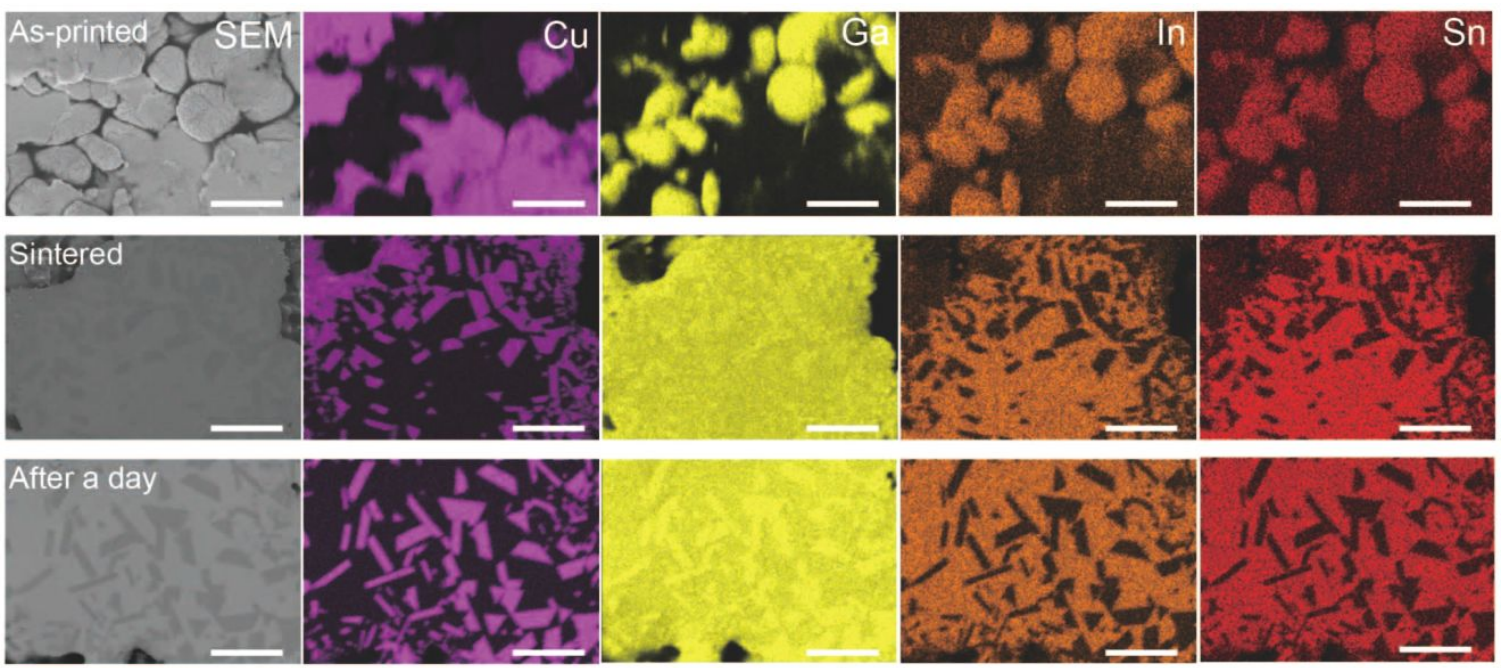

Figure S9. SEM image along with energy-dispersive X-ray spectroscopy (EDS) elemental maps of the printed composite $(\phi=\mathbf{0 . 1})$. Scale bars are $10 \mu \mathrm{m}$. After the short treatment in acid fume, the sintering reaction proceeds with the dissolution of $\mathrm{Cu}$ nanoflakes in liquid metal, followed by gradual precipitation of intermetallic compound crystals. As a result, fine crystals of intermetallic compounds are initially concentrated at the original locations of $\mathrm{Cu}$ nanoflakes.
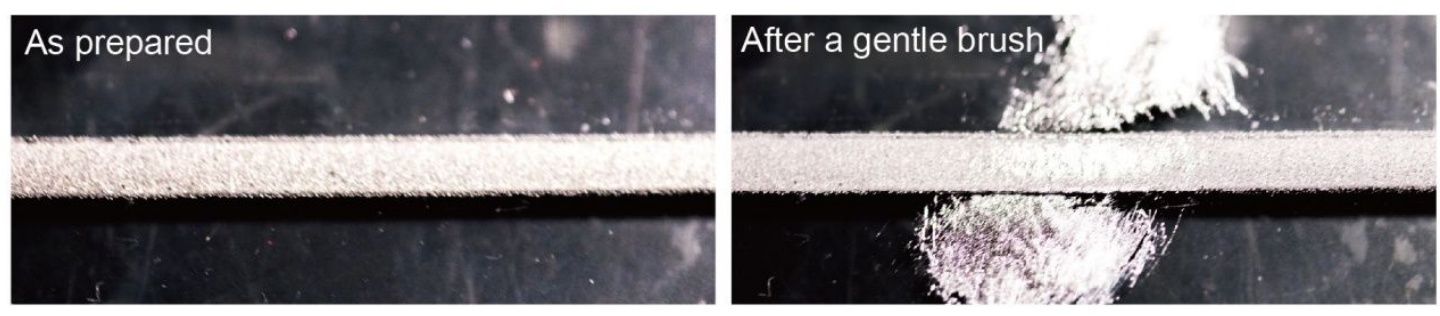

Figure S10. Scratch test for a chemically sintered feature. The sintered conductor is easily smeared by a gentle brush as a result of its liquid-state nature. 


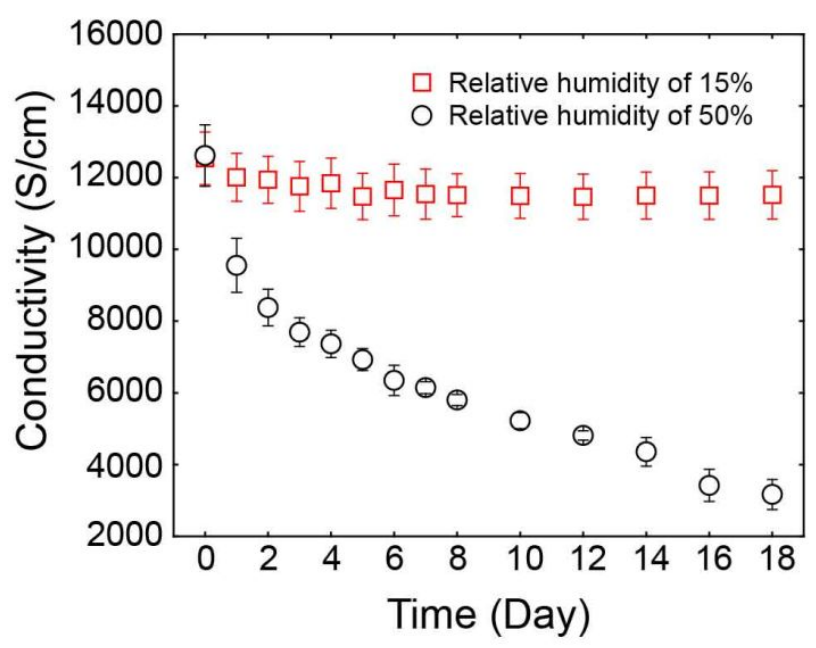

Figure S11. Long-term stability test for the chemically sintered composites. The surface oxide layer is sufficient to passivate the sintered conductor under dry air condition. Large drop in the conductivity occurs during prolonged storage in humid air (relative humidity $\sim 50 \%$ ) as the signature of chemical corrosions.

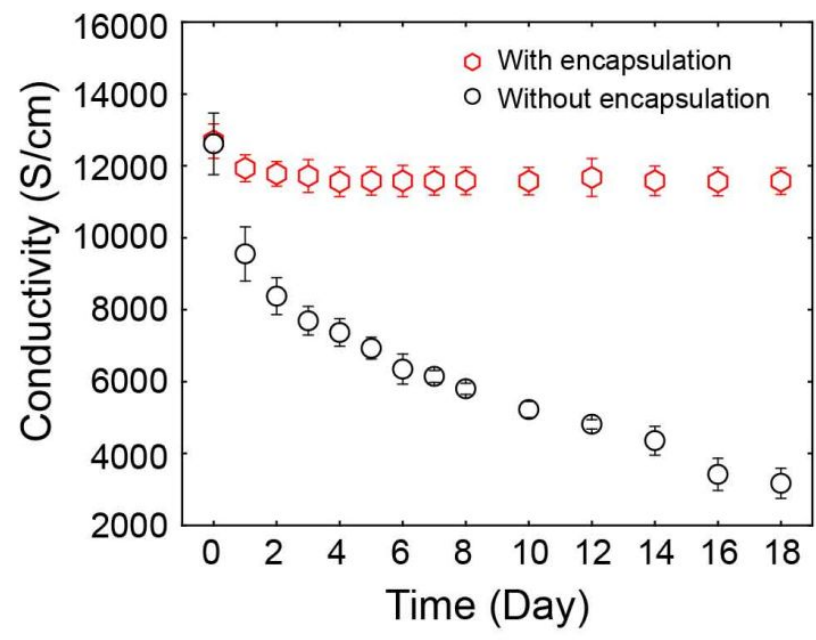

Figure S12. Influence of the encapsulation on the long-term storage stability of chemically sintered composites. Gradual decrease in the conductivity of the conductive feature is observed during prolonged storage in humid environment (relative humidity $50 \%$ ) as the result of chemical corrosions. In contrast, a $600-\mu \mathrm{m}$ thick SIS elastomeric encapsulation effectively boosts the stability to guarantee long-term storage in humid air. 


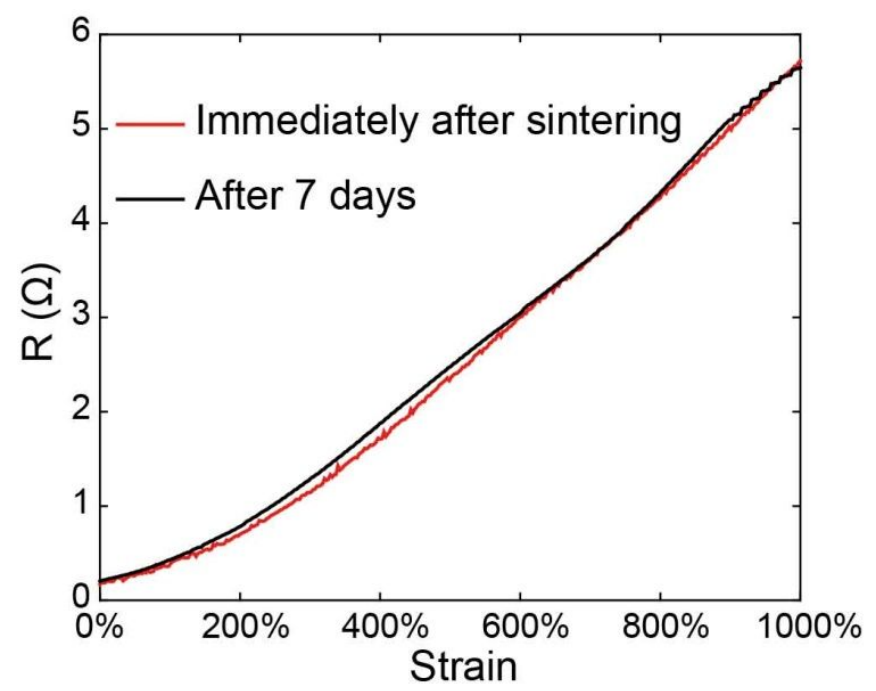

Figure S13. Influence of the storage time on the deformability of sintered composite $(\phi=\mathbf{0 . 1})$. The dependence of the resistance versus tensile strain shows negligible variation after one week, which verifies the stability of sintered conductor for long-term applications.

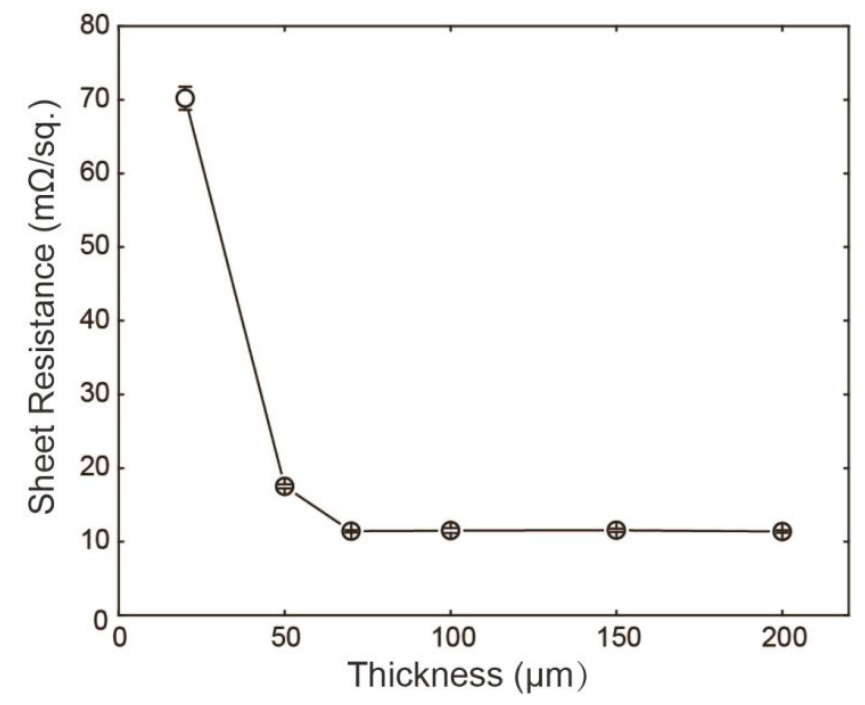

Figure S14. Sheet resistance of chemically sintered composite $(\phi=0.1)$ with different thickness. The sheet resistance shows initial decrease and gradual stabilization with increased film thickness. The thickness limit of the chemical sintering approach is determined as $70 \mu \mathrm{m}$ likely due to the finite diffusion depth of the acid vapor. Notice that 
the duration for the exposure to acid fume is fixed at $45 \mathrm{~s}$ without thorough optimization for each thickness.

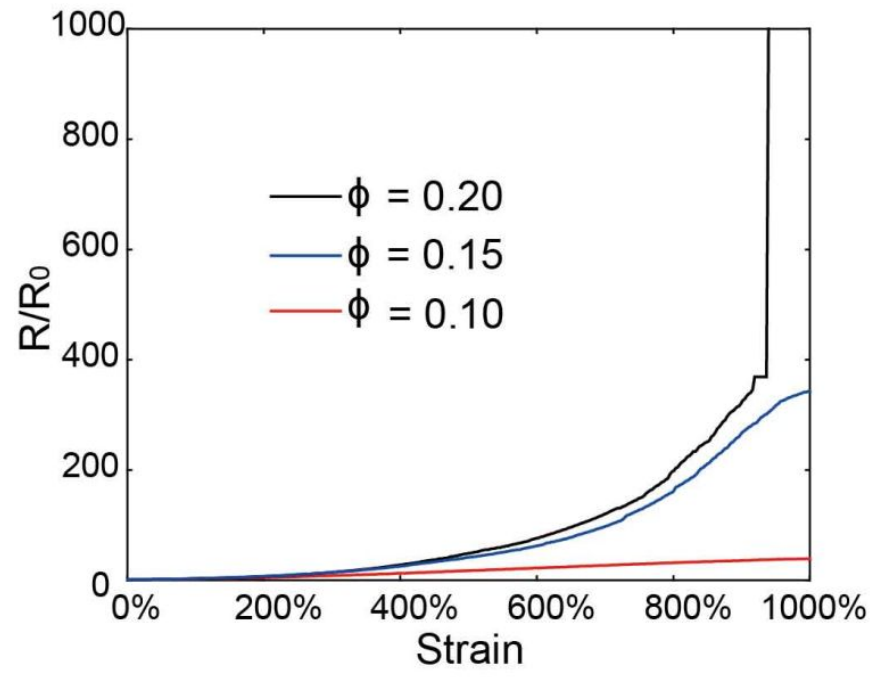

Figure S15. Change in resistance with respect to tensile strain for printed composites containing different amount of large $\mathrm{Cu}$ nanoflakes.

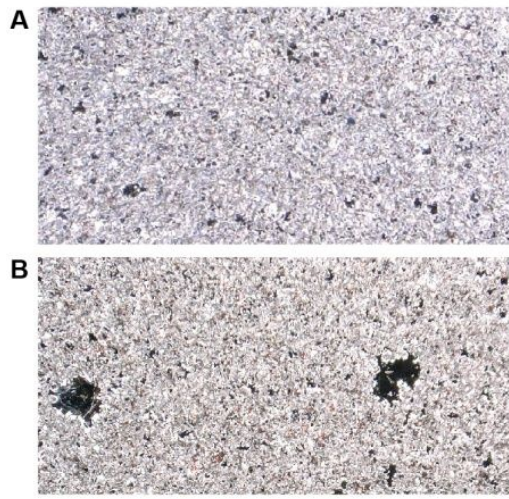

strain $=0 \%$

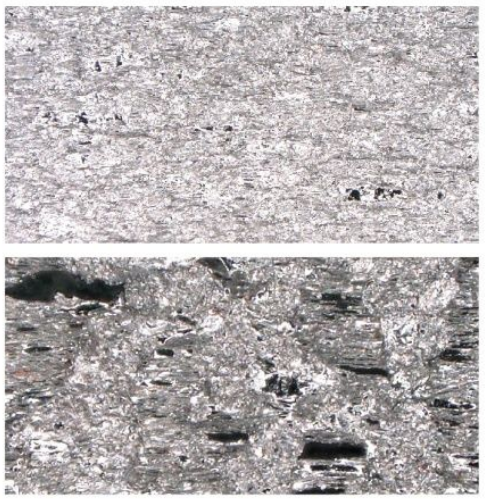

strain $=300 \%$

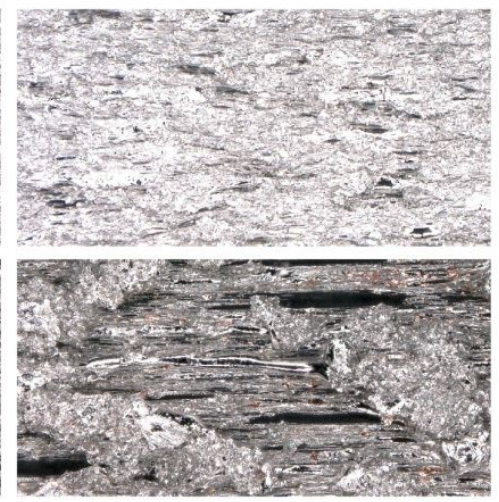

strain $=1000 \%$

Figure S16. Structural evidence for the change in the deformability of sintered conductors with different amount of large $\mathrm{Cu}$ nanoflakes. (A) Optical microscope images of sintered composites $(\phi=0.1)$ at $0 \%, 300 \%$ and $1000 \%$ strains. (B) Optical microscope images of sintered composites $(\phi=0.15)$ at $0 \%, 300 \%$ and $1000 \%$ strains. 
The dimension of each image is $1.4 \mathrm{~mm} \times 0.7 \mathrm{~mm}$.
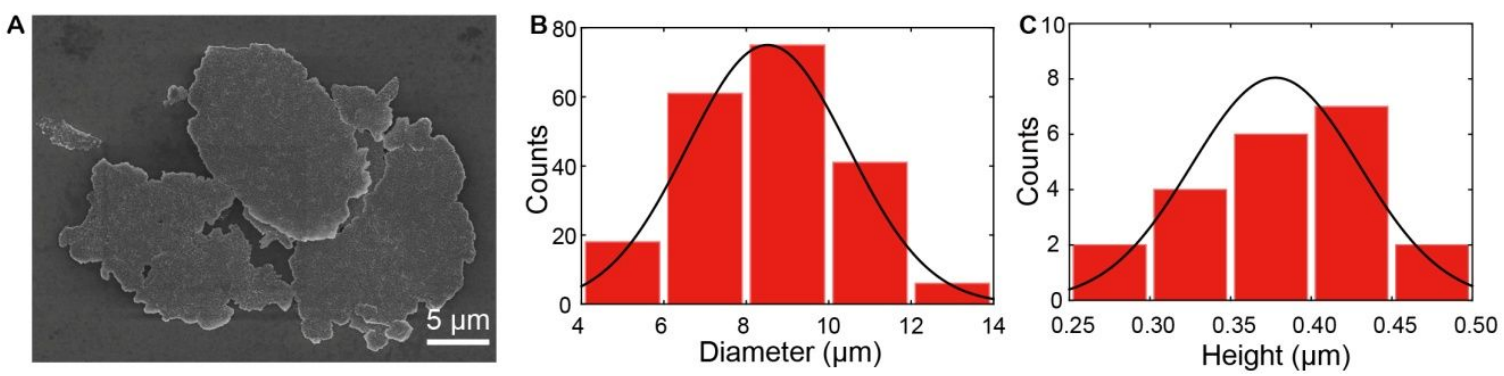

Figure S17. Structural characterizations on large Cu nanoflakes. (A) SEM image of large $\mathrm{Cu}$ nanoflakes. (B) Histogram of diameter distribution according to SEM images. (C) Histogram of thickness distribution based on laser profile measurements. Gaussian fits to the distributions (smooth curves) yield the diameter of $8.0 \pm 2.0 \mu \mathrm{m}$ and the thickness of $0.38 \pm 0.05 \mu \mathrm{m}$.
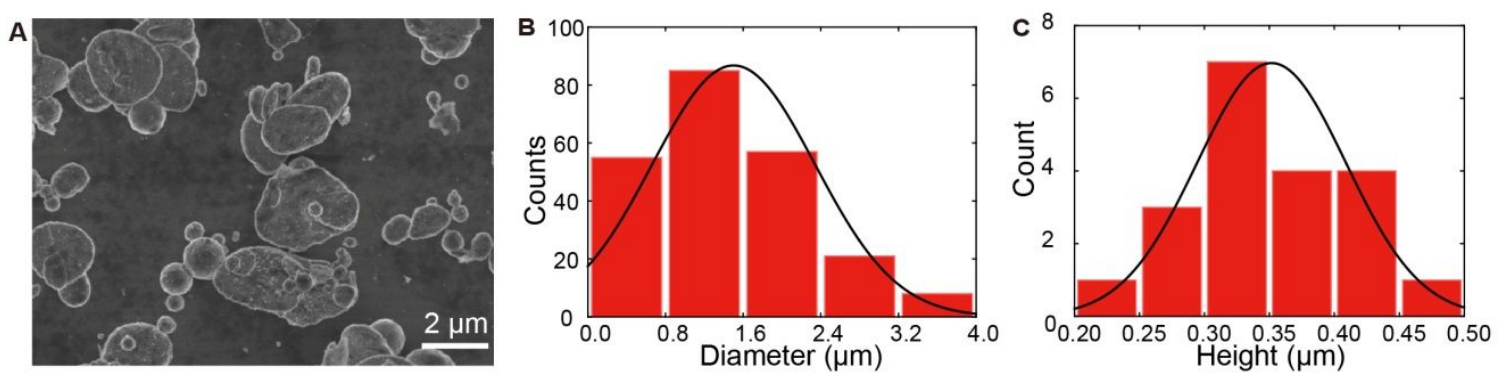

Figure S18. Structural characterizations on small Cu nanoflakes. (A) SEM image of small $\mathrm{Cu}$ nanoflakes. (B) Histogram of diameter distribution according to SEM images. (C) Histogram of thickness distribution based on laser profile measurements. Gaussian fits to the distributions (smooth curves) yield the diameter of $1.50 \pm 0.86 \mu \mathrm{m}$ and the thickness of $0.35 \pm 0.05 \mu \mathrm{m}$.
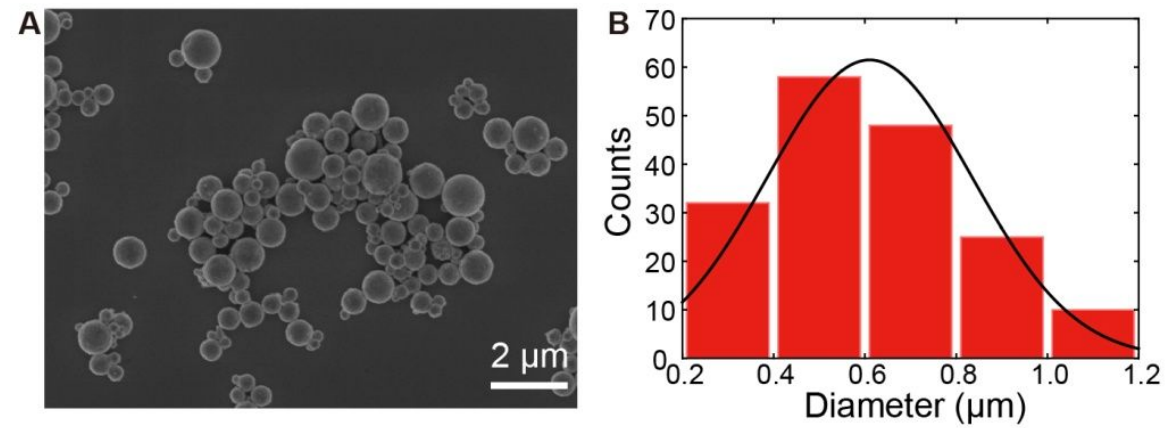
Figure S19. Structural characterizations on Cu nanoparticles. (A) SEM image of $\mathrm{Cu}$ nanoparticles. (B) Histogram of diameter distribution according to SEM images.

Gaussian fits to the distributions (smooth curves) yield the diameter of $0.60 \pm 0.22 \mu \mathrm{m}$.
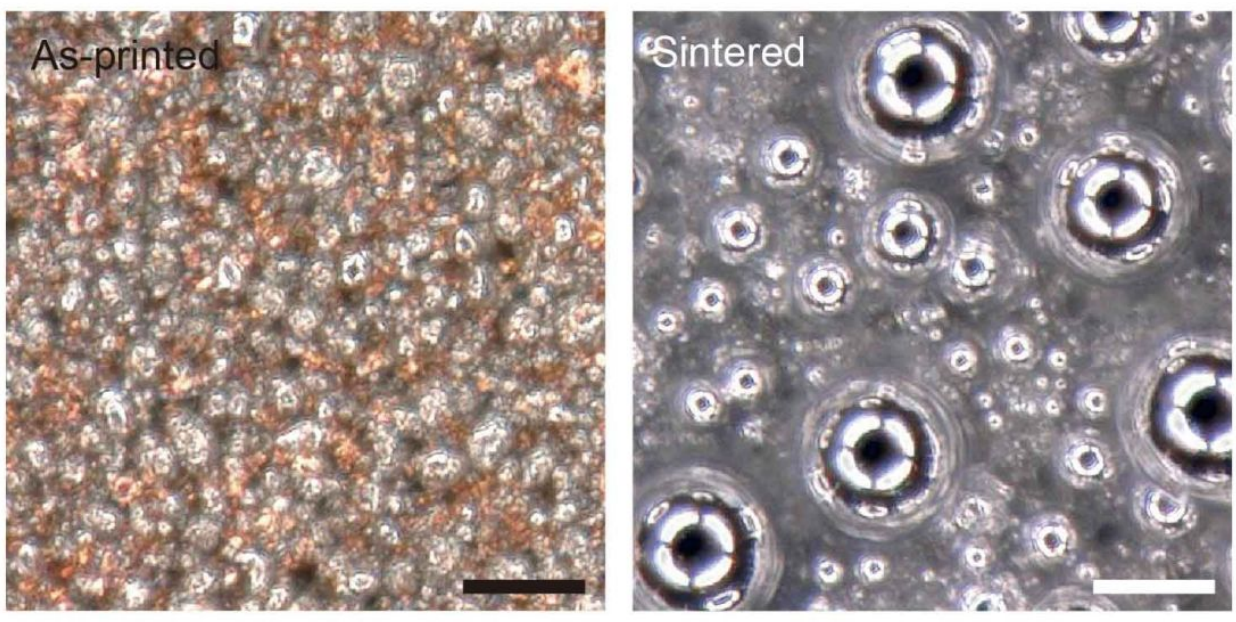

Figure S20. Optical microscopy images of the elastic conductor containing small $\mathrm{Cu}$ nanoflakes before and after chemical sintering $(\phi=0.1)$. Scale bars: $20 \mu \mathrm{m}$.
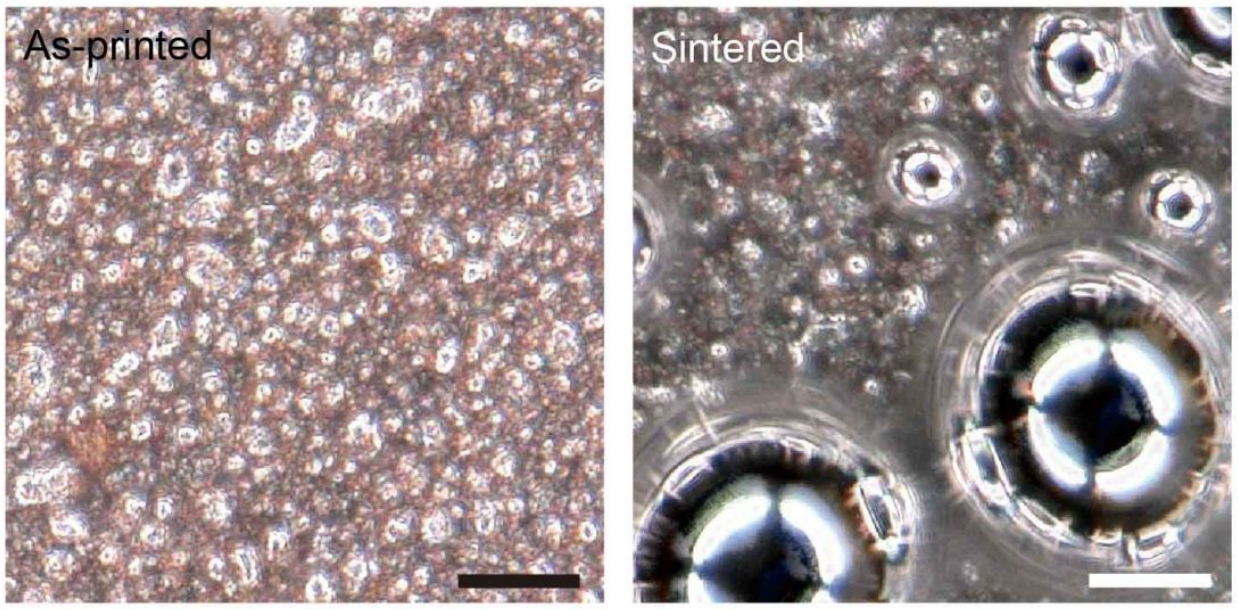
Figure S21. Optical microscopy images of the elastic conductor containing $\mathbf{C u}$ nanoparticles before and after chemical sintering $(\phi=0.1)$. Scale bars: $20 \mu \mathrm{m}$.

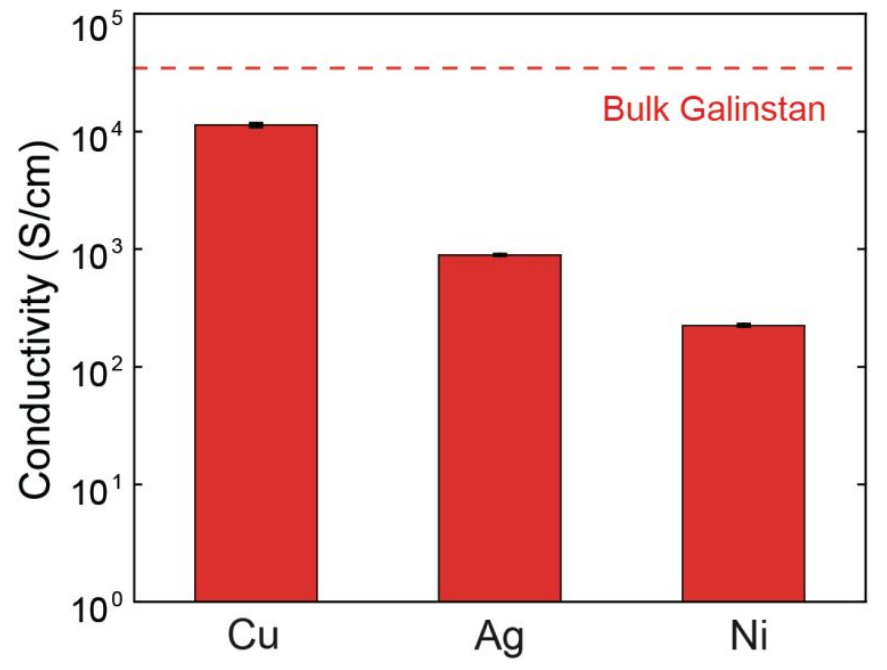

Figure S22. Electrical conductivity of sintered conductor containing different metal nanoflakes $(\phi=\mathbf{0 . 1})$. The conductivity of bulk Galinstan is marked for comparison. The diameters of $\mathrm{Cu}, \mathrm{Ag}$, and Ni nanoflakes are $8.0 \pm 2.0 \mu \mathrm{m}, 7.9 \pm 2.7 \mu \mathrm{m}$ and $9.4 \pm 2.4 \mu \mathrm{m}$, respectively. Notice that the formed intermetallic compounds instead of pristine metallic additives contribute to the conductivity of sintered conductors. ${ }^{1-2}$ The overall trend in conductivity is consistent with previous studies on Ga-based liquid metal alloys. ${ }^{1,3-5}$ 

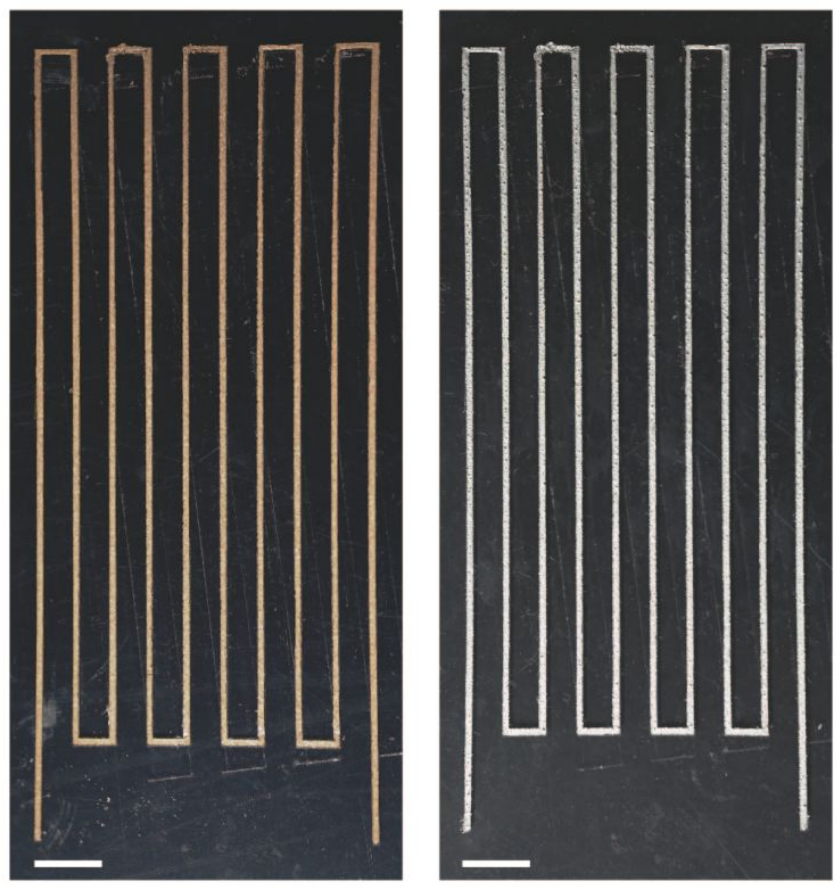

Figure S23. Optical images of the pattern design of the epidermal heater with straight wires before (left) and after (right) the chemical sintering procedure. Scale bars: $1 \mathrm{~cm}$.
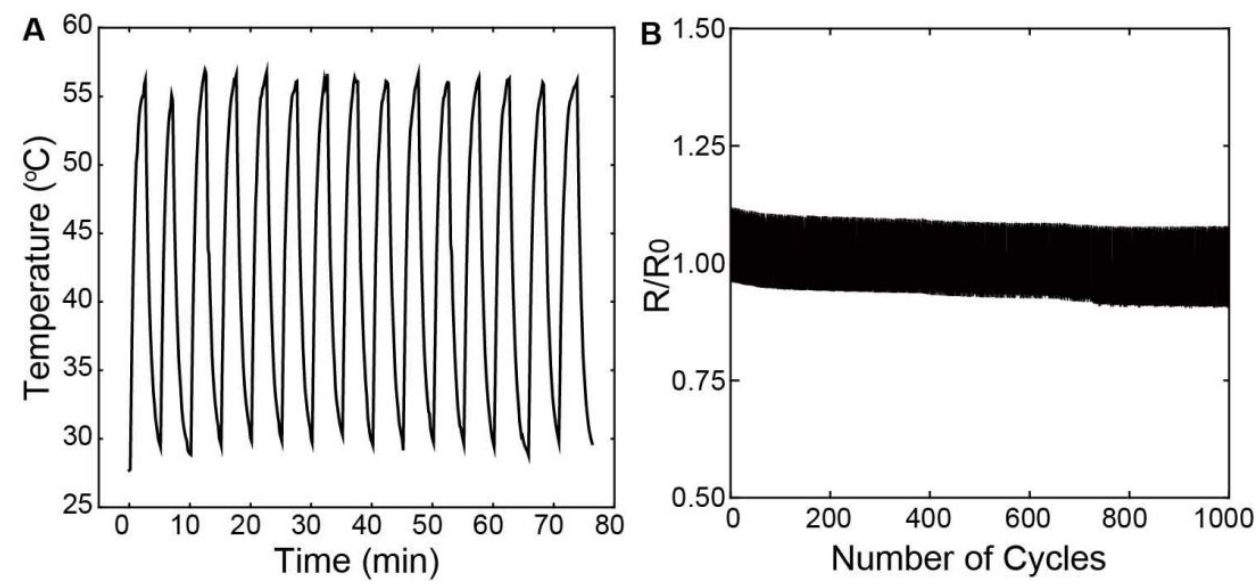

Figure S24. Characterizations on the stability of the stretchable serpentine mesh heater. (A) Temperature responses to on/off voltage cycles with an amplitude of $2.0 \mathrm{~V}$. (B) Evolution of the normalized resistance over 1000 stretch-relaxation cycles to $60 \%$ strain. 


\section{Supporting Discussions}

\section{Discussion S1. Dependence of the electrical conductivity $\sigma$ on the concentration of}

large Cu nanoflakes. The electrical properties of sintered conductor follow the percolation theory. The relationship between the electrical conductivity $\sigma$ and filler concentrations of the $\mathrm{Cu}$ nanoflakes is described by

$\sigma=\sigma_{0}\left(\phi-\phi_{\mathrm{c}}\right)^{\mathrm{s}}$

where $\sigma_{0}$ is a pre-exponential factor, $\phi_{\mathrm{c}}$ the percolation threshold, and $s$ the percolation critical exponent. Our experimental data are consistent with the predicted behavior theory with fitting parameters of $\phi_{\mathrm{c}}=0.023, \mathrm{~s}=1.36$, and $\sigma_{0}=3.38 \times 10^{5} \mathrm{~S} \mathrm{~cm}^{-1}$. The low percolation threshold verifies $\mathrm{Cu}$ nanoflakes as highly effective additive to form the conducting network.

\section{Supporting Videos}

Video S1. The operation of a stretchable ribbon cable as the interface between a LED matrix display and a microcontroller. LED matrix exhibits stable operation with dynamically refreshed luminous patterns while the cable is repetitively stretched.

\section{Supporting References}

(1) Wu, Y. h.; Deng, Z. f.; Peng, Z. f.; Zheng, R. m.; Liu, S. q.; Xing, S. t.; Li, J. y.;

Huang, D. q.; Liu, L. A Novel Strategy for Preparing Stretchable and Reliable Biphasic

Liquid Metal. Adv. Funct. Mater. 2019, 29, No. 1903840.

(2) Liu, S.; Sweatman, K.; McDonald, S.; Nogita, K. Ga-Based Alloys in Microelectronic

Interconnects: A Review. Materials 2018, 11, No. 1384.

(3) Tavakoli, M.; Malakooti, M. H.; Paisana, H.; Ohm, Y.; Green Marques, D.; Alhais

Lopes, P.; Piedade, A. P.; de Almeida, A. T.; Majidi, C. Egain-Assisted 
Room-Temperature Sintering of Silver Nanoparticles for Stretchable, Inkjet-Printed, Thin-Film Electronics. Adv. Mater. 2018, 30, No. 1801852.

(4) Tang, J.; Zhao, X.; Li, J.; Guo, R.; Zhou, Y.; Liu, J. Gallium-Based Liquid Metal Amalgams: Transitional-State Metallic Mixtures (Transm²ixes) with Enhanced and Tunable Electrical, Thermal, and Mechanical Properties. ACS Applied Materials \& Interfaces 2017, 9, 35977-35987.

(5) Guo, R.; Wang, X.; Chang, H.; Yu, W.; Liang, S.; Rao, W.; Liu, J. Ni-Gain Amalgams Enabled Rapid and Customizable Fabrication of Wearable and Wireless Healthcare Electronics. Adv. Eng. Mater. 2018, 20, No. 1800054. 